\title{
Phénotype des cellules musculaires lisses artérielles et athérosclérose
}

La prolifération, la migration et la dédifférenciation des cellules musculaires lisses jouent probablement un rôle essentiel dans la formation de la plaque athéromateuse. Les causes déclenchant ces phénomènes sont multiples. Des facteurs libérés par les plaquettes et les macrophages seraient importants dans la prolifération et la migration de ces cellules. De plus, l'altération des cellules endothéliales supprimerait un contrôle négatif de la prolifération et de la différenciation des cellules musculaires lisses. Des phénomènes immunologiques (présence de lymphocytes $\mathbf{T}$ activés et libération d'interféron) pourraient également intervenir.

\section{Laura Rubbia Giulio Gabbiani}

\footnotetext{
ADRESSE

L. Rubbia: assistante de recherche. G. Gabbiani : professeur et directeur intérimaire. Département de pathologie, université de Genève, CMU, l, rue Michel-Servet, 1211 Genève 4, Suisse.
}

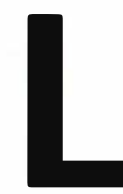

a plaque athéromateuse est constituée d'une accumulation de cellules, de matériel extracellulaire (notamment de collagène et de différents lipides) et de matériel nécrotique dans l'intima artériel. La proportion de ces composants est influencée par plusieurs facteurs tels que les mécanismes pathogéniques et l'âge de la lésion, mais, dans tous les cas, des cellules musculaires lisses (CML) provenant de la média sont présentes, souvent de façon prédominante, dans la plaque, surtout au cours des phases initiales de son développement. Les CML sont responsables de la synthèse de matériel extracellulaire, tel le collagène, et en partie de l'accumulation des lipides dans l'intima; ces dépôts provoquent à long terme le rétrécissement de la lumière vasculaire et les complications thrombotiques, par exemple l'infarctus du myocarde.

\section{Plaque d'athérome et prolifération des cellules musculaires lisses}

Les théories actuellement les plus couramment acceptées pour expliquer l'accumulation des CML au cours de la formation de la plaque suggèrent qu'elles prolifèrent et migrent vers l'intima après une disparition ou une dysfonction des cellules endothéliales, ce qui permet l'adhésion des plaquettes à la membrane basale sous-jacente d'une part et/ou la pénétration des macrophages d'autre part[1]. Ces deux types de cellules fabriquent des facteurs de croissance qui sont aussi chimiotactiques pour les CML[1] et qui seraient donc responsables des premières étapes de la formation de la plaque. Ces théories s'appuient sur des modèles expérimentaux in vivo et ex vivo; cependant, on n'a jamais pu démontrer directement chez l'homme que la plaque athéromateuse était précédée de lésions vasculaires franches ou d'un dysfonctionnement de la couche endothéliale, et cela pour des raisons techniques évidentes. De la même façon, il est difficile d'établir fermement chez l'homme l'existence d'une multiplication des CML au cours de la formation de la plaque, qui doit cependant être un des facteurs importants de sa formation. Toutefois, d'autres critères décrits récemment permettent d'évaluer l'état fonctionnel des CML dans la média 


\section{RÉFÉRENCES}

1. Ross R. The pathogenesis of atherosclerosis : an update. N Engl J Med 1986 ; 314 : 488500 .

2. Campbell GR, Campbell JH, Manderson JA, Horrigan S, Rennick RE. Arterial smooth muscle : a multifunctional mesenchymal cell. Arch Pathol Lab Med 1988; 112 : 977-86.

3. Gabbiani G, Kocher O, Bloom WS, Vandekerckhove J, Weber K. Actin expression in smooth muscle cells of rat aortic intimal thickening, human atheromatous plaque, and cultured rat aortic media. J Clin Invest $1984 ; 73: 148-52$.

4. Kocher O, Gabbiani G. Cytoskeletal features of normal and atheromatous human arterial smooth muscle cells. Hum Pathol $1986 ; 17: 875-80$

5. Rungger-Brändle E, Gabbiani G. The role of cytoskeletal and cytocontractile elements in pathologic processes. Am J Pathol 1983; 110 : 361-92.

6. Vandekerckhove J, Weber K. Actin typing on total cellular extracts. A highly sensitive protein-chemical procedure able to distinguish different actins. Eur J Biochem 1981; $113: 595-603$.

7. Skalli O, Vandekerckhove J, Gabbiani G Actin-isoform pattern as a marker of normal or pathological smooth-muscle and fibroblastic tissues. Differentiation 1987 ; 33 : 232-8.

8. Gabbiani G, Schmid E, Winter S, et al. Vascular smooth muscle cells differ from other smooth muscle cells : predominance of vimentin filaments and a specific $\alpha$-type actin. Proc Natl Acad Sci USA 1981 ; 78 : 298-

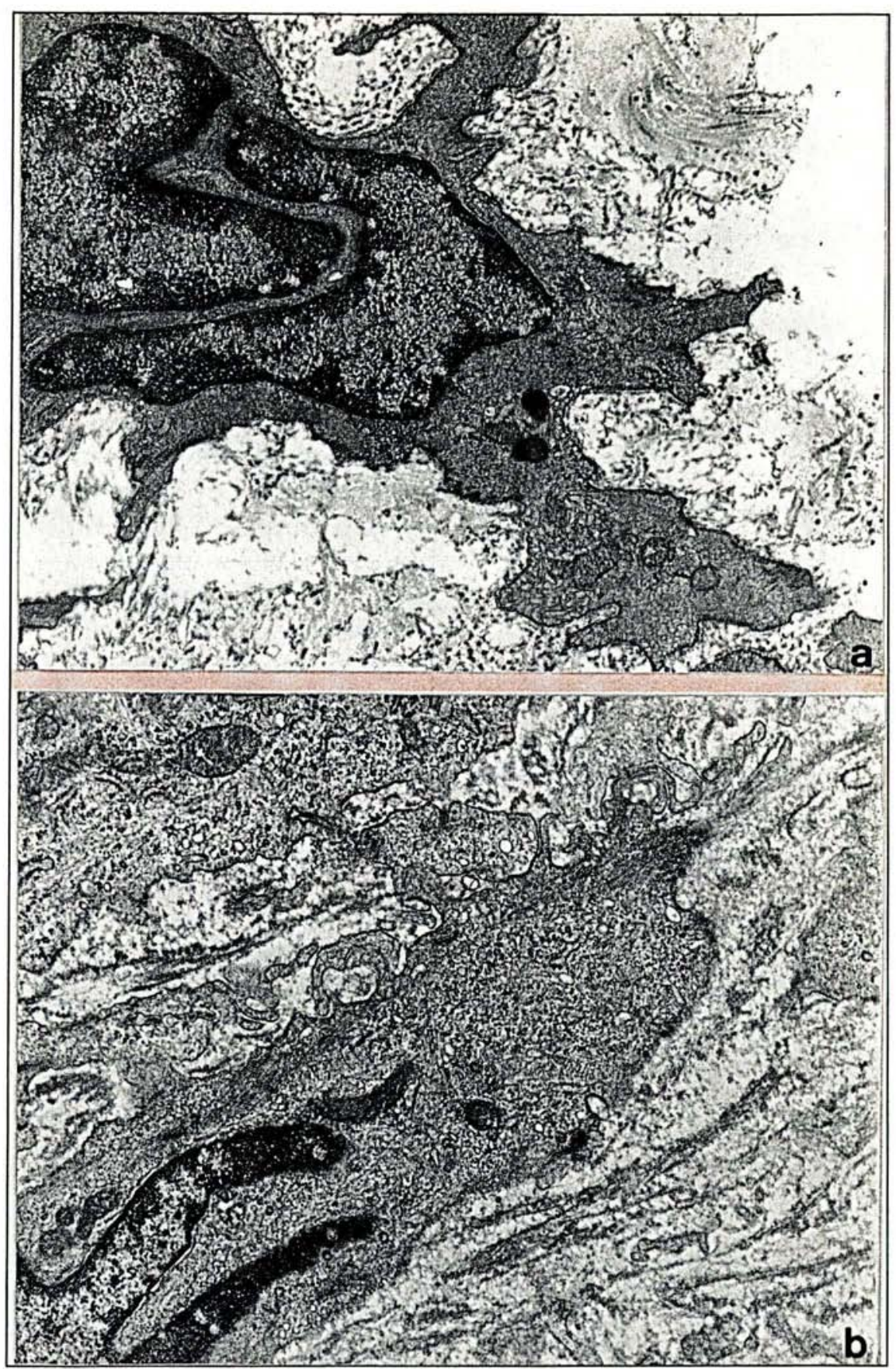

$\Delta$

Figure 1. Micrographies électroniques de cellules musculaires lisses CML provenant de la média aortique de rat adulte (a) et de l'épaississement intimal produit par lésion endothéliale au moyen d'un cathéter à ballonet (b). La CML en (a) a un phénotype différencié et « contractile», avec d'abondants faisceaux de microfilaments cytoplasmiques. La CML en (b) contient un nombre inférieur de microfilaments; le réticulum endoplasmique est bien développé, ceci donnant un aspect dédifférencié "synthétique ». ( 10500 ; figure 1a tirée de Kocher 0 , et al., Lab Invest 1984; 50: 645, avec la permission de l'éditeur.) 
et dans l'intima, et de donner ainsi une indication de la progression de la maladie athéromateuse.

\section{Modification des cellules musculaires lisses et athérome}

Les modifications qualitatives des CML au cours du développement de la plaque d'athérome peuvent être appréciées selon des critères morphologiques et biochimiques : les critères morphologiques sont basés sur l'observation que les CML normales de la média contiennent des quantités abondantes de microfilaments contractiles, tandis que les cellules de la plaque athéromateuse expérimentale et humaine sont caractérisées par une diminution des microfilaments et une augmentation du réticulum endoplasmique lisse et rugueux dans leur cytoplasme[2]. On a proposé d'appeler ces cellules riches en réticulum endoplasmique «synthétiques », par opposition aux CML présentes dans la média qui sont qualifiées de «contractiles»[2] (figure 1). L'aspect ultrastructurel «synthétique » des CML est aussi observé chez l'embryon et le fotus par rapport à l'adulte. Les critères biochimiques sont basés sur l'observation que les CML de la plaque perdent partiellement, comparativement aux CML de la média, certaines protéines de structure qui constituent aussi des marqueurs de différenciation[3, 4]. Ces protéines sont : la desmine, composant des filaments intermédiaires (une des structures cytosquelettiques avec les microtubules et les microfilaments [voir plus loin]), et l'isoforme $\alpha$-musculaire lisse de l'actine, la protéine principale des microfilaments contractiles.

Le cytosquelette de la cellule musculaire lisse. Pour mieux comprendre la signification de ces changements, nous faisons ici un bref rappel des caractéristiques cytosquettiques de la CML de la paroi artérielle. Le cytosquelette cellulaire est composé de trois structures morphologiquement et fonctionnellement différentes, $\mathrm{m} / \mathrm{s} n^{\circ} 6$ vol. 5 , juin 89 toutes organisées sous forme filamenteuse: les microfilaments, les microtubules, et les filaments intermédiaires (pour revue, voir [5]).

Les microfilaments, d'un diamètre de $6 \mathrm{~nm}$ (nanomètres) environ, sont composés essentiellement d'actine, protéine globulaire qui polymérise sous forme filamenteuse. L'actine est accompagnée de nombreuses autres protéines qui servent à moduler l'organisation des microfilaments, en réglant par exemple sa polymérisation. Ainsi certaines protéines (telle la profiline) gardent l'actine sous forme globulaire, d'autres (telle la filamine) favorisent le maintien des microfilaments, et enfin d'autres (telle la gelsoline) coupent les filaments en portions plus courtes de façon à faciliter la dynamique de la réorganisation de ces derniers. L'actine est la protéine contractile quantitativement la plus importante dans les CML et son interaction spécifique avec la myosine provoque leur contraction.

L'actine est présente dans toutes les cellules eucaryotes et possède une structure primaire très conservée au cours de la phylogenèse. Cependant, chez les vertébrés supérieurs, elle est exprimée en six isoformes de même poids moléculaire, mais qui peuvent être séparées selon leur point isoélectrique par une électrophorèse en deux dimensions [6] et sont désignées par les lettres grecques $\alpha$, B et $\gamma$. En position $\alpha$ migrent trois isoformes appelées $\alpha$-cardiaque, $\alpha$-squelettique et $\alpha$-musculaire lisse, car elles sont présentes de façon prédominante respectivement dans ces types cellulaires. Il faut noter que l'actine $\alpha$ musculaire lisse est présente surtout dans les CML vasculaires [7]. En position $\beta$ migre une seule isoforme appelée cytoplasmique car elle est présente dans toutes les cellules. Enfin, en position $\gamma$ migrent deux isoformes, l'une cytoplasmique et l'autre présente de façon prédominante dans les CML parenchymateuses, telles que celles de l'intestin, des bronches et de l'appareil génital. Les fonctions exactes de ces isoformes ne sont pas connues, mais il est bien établi que leur apparition spécifique correspond à la différenciation des cellules musculaires. Plusieurs expériences ont aussi suggéré que la spécificité des isoformes d'ac- tine est en relation avec l'activité fonctionnelle de chaque type de cellules musculaires.

Les microtubules sont des tubes creux d'environ $22 \mathrm{~nm}$ de diamètre composés essentiellement de tubuline, et d'une série de protéines associées qui règlent la polymérisation de cette dernière. Les microtubules sont en relation avec plusieurs fonctions cellulaires importantes telles que le transport cytoplasmique et la mitose, mais ne seront pas traités lors de cet exposé.

Les filaments intermédiaires enfin sont ainsi appelés parce que leur diamètre d'environ $11 \mathrm{~nm}$ est intermédiaire entre celui des microfilaments et celui des microtubules. Ces filaments sont les plus insolubles du cytoplasme et donc sont considérés comme le véritable élément cytosquelettique de la cellule. Même si les filaments intermédiaires des différentes cellules ont une morphologie semblable, ils sont composés de protéines différentes selon l'origine embryologique de la cellule [5]. Ainsi, ils constituent des marqueurs de différenciation très étudiés. Les CML contiennent essentiellement des filaments intermédiaires qui sont des homopolymères de desmine, à l'exception des CML vasculaires qui sont hétérogènes, pouvant contenir soit des filaments intermédiaires composés exclusivement de vimentine, la protéine typique des cellules mésenchymateuses (par exemple fibroblastes et leucocytes), soit des hétéropolymères de vimentine et de desmine [8].

L'étude des composants cytosquelettiques a démontré l'existence de changements caractéristiques dans les CML de la plaque athéromateuse expérimentale et humaine comparativement aux cellules de la média artérielle. Ces changements sont: une disparition de la desmine, au moins au cours des phases initiales [4] (figure 2), et un changement de la proportion des isoformes d'actine, allant d'une prédominance de l'isoforme $\alpha$ dans les CML de la média à une prédominance de l'isoforme $\beta$ dans les CML de la plaque [3] (figure 3). Ces modifications suivent de façon caractéristique l'évolution inverse de celle observée au cours du développement, ou les CML acquièrent graduellement la 

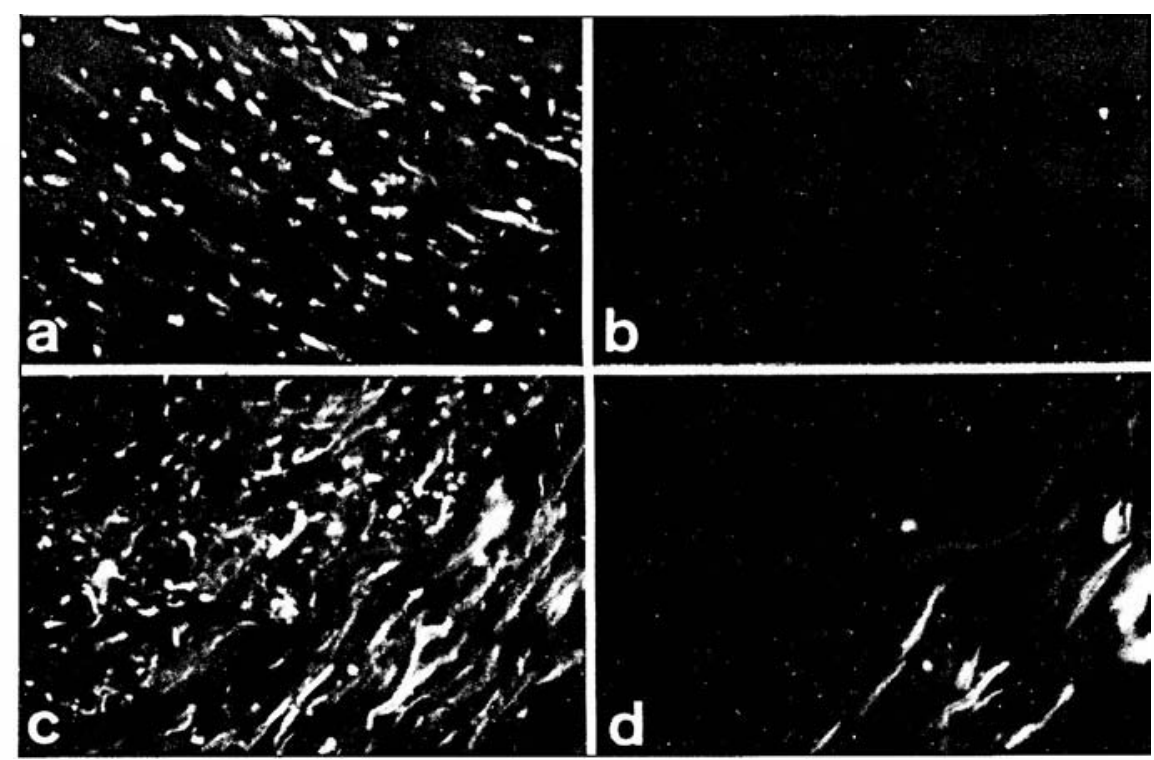

$\Delta$

Figure 2. Double immunofluorescence pour la vimentine $(a, c)$ et la desmine $(b, d)$ dans des plaques athéromateuses humaines fibreuses isolées d'une artère fémorale $(a, b)$ et d'une artère coronaire (c, d). Une zone de média est visible dans la coupe d'artère coronaire en-dessous de la membrane élastique interne faiblement autofluorescente (d). Toutes les cellules musculaires lisses CML de la plaque $(a, c)$ et de la média (c) expriment la vimentine. La desmine est absente dans les cellules de la plaque $(\boldsymbol{b}, \boldsymbol{d})$ et présente dans plusieurs cellules de la média (d). (x 400 ; tirée de Kocher O, Gabbiani G, Hum Pathol $1986 ; 17: 875$, avec la permission de l'éditeur).

9. Kocher O, Skalli O, Cerutti D, Gabbiani F, Gabbiani G. Cytoskeletal features of rat aortic cells during development. An electron microscopic, immunohistochemical, and biochemical study. Cir Res $1985 ; 56: 829-38$.

10. Clowes AW, Clowes MM. Kinetics of cellular proliferation after arterial injury. II. Inhibition of smooth muscle growth by heparin. Lab Invest 1985 ; 52 : 611-16.

11. Hansson GK, Jonasson L, Lojsthed B Stemme S, Kocher O, Gabbiani G. Localization of Tlymphocytes and macrophages in fibrous and complicated human atherosclerotic plaques. Atherosclerosis $1988 ; 72$ : 135-41.

12. Rubbia L, Sappino PA, Hansson HK, Gabbiani G. Action of different cytokines on actin isoforms expression on fibroblasts and arterial smooth muscle celles in vitro. Experientia 1989 ; 45 : A 49.

13. Clowes AW, Karnovsky MJ. Suppression by heparin of smooth muscle cell proliferation in injured arteries. Nature 1977; 265: 625-6.

14. Clowes AW, Clowes MM, Kocher O, RoprazP, Chaponnier C, Gabbiani G. Arterial smooth muscle cells in vivo : relationship between actin isoform expression and mitogenesis and their modulation by heparin. $J$ Cell Biol 1988; 107 : 1939-45.

15. Majesky MW, Schwartz SM, Clowes MM, Clowes AW. Heparin regulates smooth muscle $S$ phase entry in the injured rat carotid desmine aux dépens de la vimentine et passent d'une prédominance de l'isoforme $\beta$ de l'actine chez le foetus et le nouveau-né à une prédominance de l'isoforme $\alpha$ chez l'adulte [9]. Il semble donc qu'au cours de la formation de la plaque athéromateuse les CML subissent un processus de dédifférenciation qui les rend semblables aux cellules fœtales. Nous rappelons que les cellules fœtales sont des cellules qui se répliquent activement, comme le sont probablement les cellules de la plaque, tandis que les cellules de la média artérielle adulte normale ont un taux de réplication extrêmement bas [10].
Figure 3. Séparation au moyen d'une électrophorèse en deux dimensions des isoformes d'actine présentes dans les cellules musculaires lisses CML de la média aortique (a) et d'une plaque fibreuse (b) humaines. En haut à gauche, la séparation des mêmes isoformes est effectuée au moyen d'une électrophorèse monodimensionnelle, selon le point isoélectrique. Dans la média (a), I' $\alpha$-actine musculaire lisse est l'isoforme prédominante. Dans l'épaississement intimal (b), on note une prédominance de l'isoforme $\beta$-cytoplasmique (tirée de Kocher $O$ et Gabbiani G, Hum Pathol 1986; 17: 875, avec la permission de l'éditeur). 
En résumé, il semble donc qu'au cours de la formation de la plaque athéromateuse, les CML migrent de la média vers l'intima artérielle, se multiplient et se dédifférencient.

Les phénomènes amenant à la production de fibrose et de nécrose seraient consécutifs à ces premiers événements. Il est donc important d'étudier les facteurs qui règlent la division, la migration et la différenciation des CML artérielles.

\section{Facteurs de croissance, cellules $T$ et athérome}

Comme nous l'avons dit précédemment, on considère généralement que des facteurs libérés par les plaquettes et les macrophages (essentiellement les différents types de PDGF ou platelet derived growth factor)

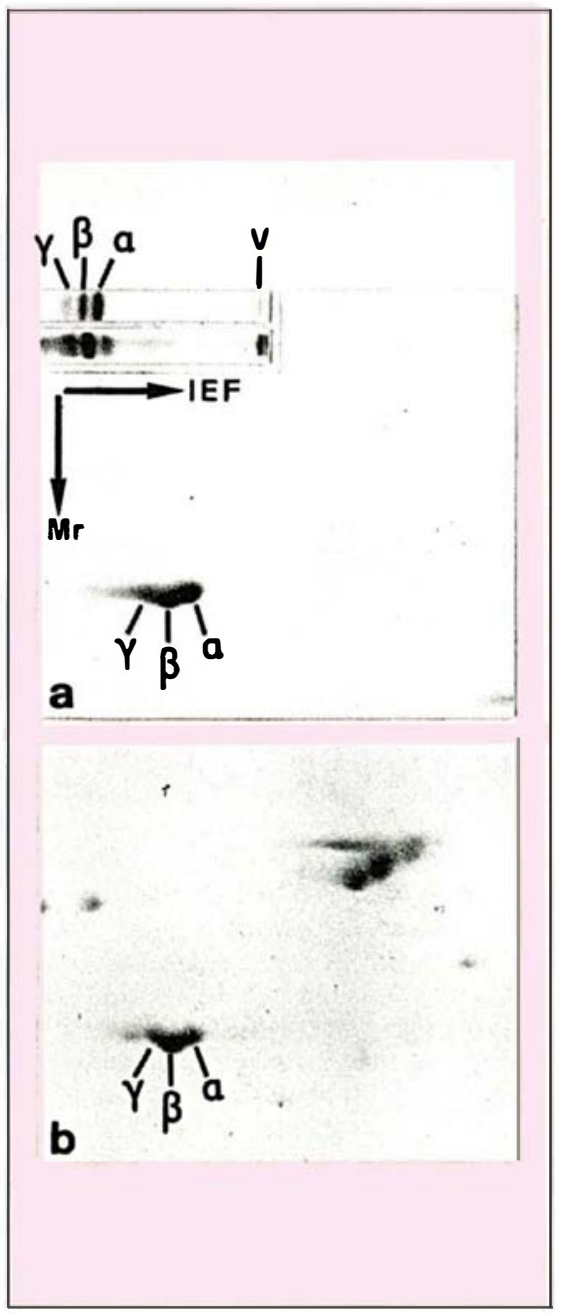

$\mathrm{m} / \mathrm{s} n^{\circ} 6 \mathrm{vol} .5$, juin 89 sont capables d'induire au moins les deux changements (migration et réplication des CML) caractéristiques du début de la formation de la plaque [1]. Il reste à savoir comment ces facteurs arrivent à influencer les CML en présence d'un endothélium morphologiquement non altéré. Il est donc important d'étudier les modifications fonctionnelles de l'endothélium en présence de facteurs prédisposant à l'athéromatose. D'autre part, des études récentes démontrent que la plaque athéromateuse contient, même précocement, une proportion importante de lymphocytes qui se sont révélés être presque exclusivement des lymphocytes $\mathrm{T}$ du type auxiliaire (helper) [11]. De plus, une proportion non négligeable de CML de la plaque expriment des molécules de classe II du complexe majeur d'histocompatibilité[11]. Cette expression, caractéristique des cellules présentant les antigènes, est généralement due à l'action de l'interféron $\gamma$ qui est un produit des lymphocytes $\mathrm{T}$ auxiliaires. Des études récentes ont aussi démontré que l'interféron $\gamma$ inhibe sélectivement la synthèse de l'ARN messager de l' $\alpha$-actine musculaire lisse dans les CML de rat et de l'homme in vitro, provoquant une diminution de la protéine correspondante et donc une dédifférenciation des CML [12]. Des phénomènes de type immunologique pourraient ainsi être impliqués dans le développement et dans l'entretien de la plaque athéromateuse; des études ultérieures seront nécessaires pour vérifier de façon plus précise cette hypothèse.

\section{Cellules endothéliales et contrôle de la multiplication des cellules musculaires lisses}

Une autre observation importante a permis d'entrevoir des mécanismes de développement de la plaque athéromateuse qui étaient totalement insoupçonnés. L'héparine, et de façon plus sélective les héparan-sulfates produits par les cellules endothéliales, inhibent la réplication des CML artérielles in vivo et in vitro[13]. De plus, l'héparine

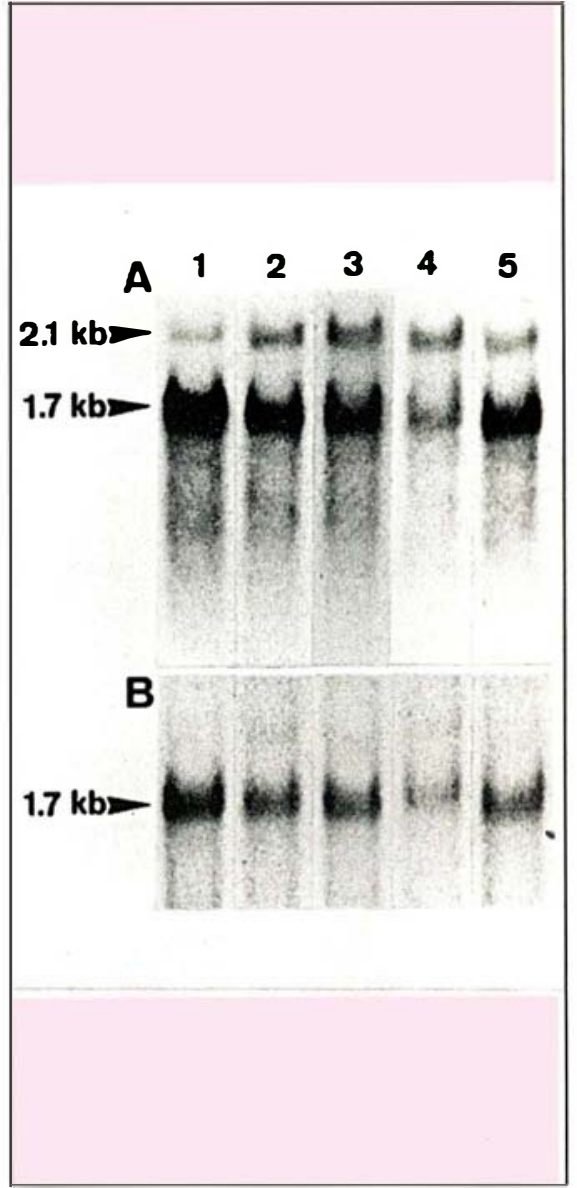

Figure 4. Autoradiographie de "Northern blots" des ARN totaux extraits d'artère carotide de rats traités ou non à l'héparine, et présentant une lésion endothéliale expérimentale. L'hybridation a été effectuée avec une sonde reconnaissant l'ensemble des messagers de l'actine (A), qui met en évidence les formes $\alpha$-musculaires $(1,7 \mathrm{~kb})$ et $\beta$-plus $\gamma$-cytoplasmiques $(2,1 \mathrm{~kb})$, et une sonde spécifique du messager de l'a-actine musculaire lisse (B). Ligne 1: carotide témoin ; lignes 2-3: carotide 24 heures après lésion chez des animaux traités avec une solution saline (2) ou l'héparine (3); lignes 4-5: carotide cinq jours après lésion, chez des animaux traités avec une solution saline contrôle (4) ou l'héparine (5). Une diminution de l'expression du messager de l' $\alpha$-actine musculaire lisse est visible 24 heures après la lésion endothéliale indépendamment du traitement à l'héparine. En revanche, une réinduction du messager pour l' $\alpha$-actine musculaire est visible seulement cinq jours après la lésion chez les animaux traités à l'héparine (tirée de Clowes AW, et al., J Cell Biol 1988; 107: 1939, avec la permission de l'éditeur). 
empêche la diminution de l' $\alpha$-actine musculaire lisse qui a 1 ieu, soit au cours de la formation in vivo d'une plaque athéromateuse expérimentale [14] (figure 4), soit dans les CML qui se répliquent in vitro sous l'action du sérum ou de facteurs de croissance (observation non publiée). Le fait que les cellules endothéliales produisent ces héparan-sulfates permet d'émettre l'hypothèse que le contrôle de la quiescence et de la différenciation des CML de la média normale est activement assuré par des substances produites par la cellule endothéliale [13-15]. En effet, la meilleure méthode expérimentale pour induire une réplication des CML chez l'animal est la destruction de la couche endothéliale par un cathéter à ballonnet. L'hypothèse d'une inhibition active de la réplication des CML est attrayante, car elle suggère des possibilités thérapeutiques, d'autant plus que l'activité antimitotique de l'héparine pour les CML n'est apparemment pas liée à son activité anticoagulante.

Il est clair que la pathogenèse de la plaque athéromateuse est multiple, mais il est aussi évident que les changements observés au niveau des CML jouent un rôle important dans sa formation et dans le développement de ses complications (figure 5, représentation schématique des facteurs influençant les CML). Il ne semble donc pas hasardeux de prévoir que des études sur la réplication, la migration et la différenciation des CML artérielles permettront de mieux comprendre les mécanismes de formation de cette lésion et d'établir des stratégies pour une approche préventive et thérapeutique efficace

\section{Remerciements}

Nous remercions le Fonds National Suisse de la Recherche Scientifique (subvention n 3.108-0.88), les éditeurs de Human Pathology, Journal of Cell Biology et Laboratory Investigation pour leur permission de reproduire les figures $1 a, 2,3$ et 4 , et Mme M.M. Rossire pour avoir tapé le manuscrit.

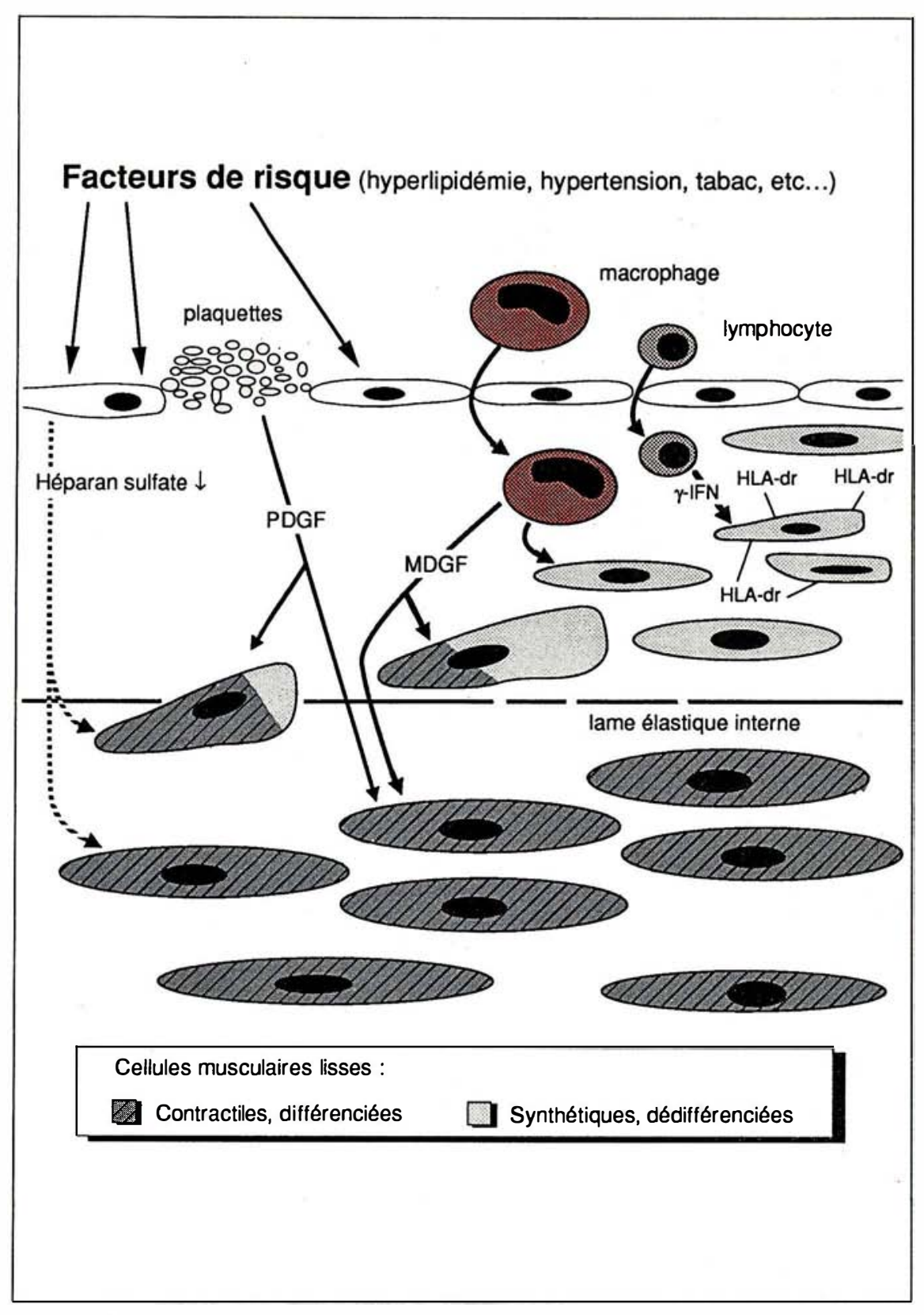

Figure 5. Représentation schématique des facteurs jouant un rôle dans le changement de phénotype des CML au cours de l'athéromatose. Les facteurs de risque produisent une perte et/ou un dysfonctionnement de l'endothélium qui entraînent l'agrégation plaquettaire et/ou la pénétration de macrophages et de lymphocytes dans I'intima. Les facteurs chimiotactiques et mitogènes (tels que le platelet derived growth factor, PDGF ou le macrophage derived growth factor, MDGF) ainsi libérés agissent sur les CML de la média qui se divisent, se dédifférencient et migrent dans l'intima. L'accumulation des CML et de leurs produits de sécrétion provoque progressivement la formation de la plaque athéromateuse. Les lymphocytes T de type auxiliaires présents dans I'intima produisent de l'interféron- $\gamma$ (IFN $\gamma$ ) qui induit lui-même l'apparition de molécules du complexe majeur d'histocompatibilité (HLA dr) en surface et une diminution de l'a-actine musculaire lisse dans les CML de la plaque. Les héparan-sulfates, produits par les cellules endothéliales, inhibent normalement la prolifération des CML et les maintiennent dans un état différencié. La diminution de ces substances sous l'action de facteurs de risque joue aussi un rôle dans la prolifération et la dédifférenciation des CML. 


\section{Summary}

Phenotype of arterial smooth muscle cells and atheromatosis During the formation of the atheromatous plaque, smooth muscle cells (SMC) migrate from the arterial media to the intima where they replicate and synthesize important amounts of extracellular material. Concurrently, SMC assume cytoskeletal features of embryonic and fetal cells such as : decrease in the amount of desmin filaments and increase in the proportion of the $\beta$-cytoplasmic isoform of actin, whereas in normal medial SMC, the $\alpha$-smooth muscle isoform of actin is predominant. The factors controlling the initiation of atheromatous plaque formation depend probably on changes in endothelial functions which allow growth factors from platelets and macrophages (essentially platelet derived growth factor) to stimulate the migration and proliferation of SMC. T helper lymphocytes (possibly through the production of $\gamma$ interferon) may participate in the maintenance of SMC dedifferentiation. Finally, heparan sulfates produced by endothelial cells may control the quiescence and differentiation of SMC, thus contributing to the normal organization of the arterial wall and, when lacking, to the formation of the atheromatous plaque.

\section{TIRÉS A PART}

G. Gabbiani.

$m / s n^{\circ} 6$ vol. 5 , juin 89
TROISIĖME WORKSHOP INTERNATIONAL DE LARTAC SUR L'EXPÉRIMENTATION THÉRAPEUTIQUE EN CANCÉROLOGIE : CONCEPTS SCIENTIFQUES, MÉTHODOLOGIE ET NOUVEAUX MÉDICAMEVIS PARIS, 19 ET 20 OCTOBRE 1989

A l'occasion de l'année Européenne d'information sur le cancer; l'Association pour la Recherche Thérapeutique Anticancéreuse (ARTAC), organise son troisième congrès, à la Maison de la Chimie, 28, rue SaintDominique, 75007 Paris, les 19 et 20 octobre prochain.

Les nouveaux concepts scientifiques issus de la recherche fondamentale permettent aujourd'hui d'espérer de nouveaux progrès thérapeutiques en cancérologie. Ces nouveaux concepts imposent de reconsidérer certains aspects de la méthodologie des essais cliniques.

Le $3^{\mathrm{e}}$ workshop de I'ARTAC est organisé par le docteur Dominique Belpomme, cancérologue à l'hôpital Bichat, Paris, président de l'ARTAC et par le docteur Michel Clavel, Cancérologue au centre Léon Bérard, Lyon, délégué pour la France de la Société Européenne d'Oncologie Médicale (ESMO).

Participeront à cette manifestation, plusieurs médecins et chercheurs de l'Organisation Européenne de Recherche et de traitement des Cancers (EORTC), du National Cancer Institute (Bethesda), de l'ESMO, ainsi que plusieurs scientifiques japonais.

Le programme couvre l'ensemble des aspects scientifiques, méthodologiques et éthiques du développement préclinique et clinique des nouveaux produits ou médicaments anticancéreux.

- La première partie du congrès est consacrée à un cours intensif de méthodologie appliquée à l'expérimentation clinique. Seront abordés: le programme d'évaluation de la recherche thérapeutique au $\mathrm{NCl}$, l'expérience de l'EORTC et du $\mathrm{NCl}$ dans le domaine du screening préthérapeutique des nouveaux produits, les aspects méthodologiques des essais de phase I, II et III, ainsi que l'utilisation des Bonnes Pratiques Cliniques en cancérologie et les questions de bioéthique.

- La seconde partie fait le point sur les nouveaux concepts biologiques utilisables en clinique. Seront tour à tour discutés les mécanismes moléculaires impliqués dans les phénomènes de cytotoxicité, de chimiorésistance, et de facilitation métastatique chimio-induite, les problèmes de différenciation cellulaire, les oncogènes et anti-oncogènes, les facteurs de croissance et hormones, les mécanismes de la résistance de l'hôte face au cancer. L'objectif essentiel de cette manifestation est de mettre à la portée des investigateurs cliniciens, les bases scientifiques indispensables à l'élaboration de nouveaux protocoles thérapeutiques, en insistant plus particulièrement sur les problèmes de transfert de concepts et de technologie, de la biologie à la clinique.

- La deuxième journée est consacrée à l'état actuel du développement des nouveaux médicaments anticancéreux, en France, en Europe et au Japon. Ce thème sera abordé avec le concours de plusieurs groupes pharmaceutiques. Au cours d'une table ronde réunissant différents représentants de l'industrie pharmaceutique et de l'administration, seront aussi évoqués les problèmes actuels concernant les nouvelles procédures européennes de mise sur le marché.

LARTAC est une association à but non lucratif, fondée en 1984 par des cancérologues, industriels et malades, dans le but de promouvoir la recherche clinique en cancérologie et plus particulièrement de concourir au développement clinique des nouveaux produits, dans le cadre d'une action de partenariat avec l'industrie. L'organisation d'un tel workshop témoigne de la vocation pédagogique de I'ARTAC.

Pour toute information concernant l'inscription au workshop et/ou I'ARTAC, contacter Mademoiselle Fabienne Caron, ARTAC, 38, rue de Silly, 92100 Boulogne/Seine. Tél. : (1) 48.25.40.09. 